\title{
Prosthetically-Driven Alveolar Reconstruction: A Case Report
}

ISSN: 2637-7764

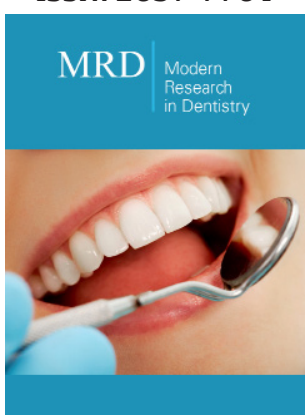

*Corresponding author: Guaracilei Maciel Vidigal Junior, Adjunct Professor, Faculty of Dentistry, Department of Clinical Integrated Procedures, Universidade do Estado do Rio de Janeiro (UERJ) Av. 28 de setembro 157, Vila Isabel, Rio de Janeiro, ZIP: 20551-030, RJ, Brazil

Submission: 此 April 24, 2020

Published:

Volume 5 - Issue 1

How to cite this article: Guaracilei Maciel Vidigal Junior, Luiz Roberto Figueiredo Dantas. Prosthetically-Driven Alveolar Reconstruction: A Case Report. Mod Res Dent. 5(1). MRD.000605.2020. DOI: 10.31031/MRD.2020.05.000605

Copyright@ Guaracilei Maciel Vidigal Junior, This article is distributed under the terms of the Creative Commons Attribution 4.0 International License, which permits unrestricted use and redistribution provided that the original author and source are credited.

\section{Guaracilei Maciel Vidigal Junior ${ }^{1 *}$ and Luiz Roberto Figueiredo Dantas ${ }^{2}$}

${ }^{1}$ Adjunct Professor, Department of Clinical Integrated Procedures, Rio de Janeiro State University (UERJ), Brazil

${ }^{2}$ Associate Researcher, Rio de Janeiro State University (UERJ), Brazil

\begin{abstract}
s
Prosthetically-driven alveolar reconstruction (PDAR) technique [1] is a method for alveolar ridge preservation/regeneration after tooth extraction without flaps, membranes or grafts with optimum aesthetic results. This case report presents a clinical situation where an upper right pre-molar exhibits an extensive buccal bone plate loss related to a late paraendodontic surgery failure. Using the PDAR technique complete bone regeneration was achieved using a minimally invasive procedure with optimum aesthetic results.
\end{abstract}

Keywords: Alveolar bone preservation/regeneration; Extraction socket; Coagulum; Fixed provisional prosthesis

\section{Introduction}

Prosthetically-driven alveolar reconstruction (PDAR) technique [1] is a method for alveolar ridge preservation/regeneration after tooth extraction and is indicated for extraction sockets with the absence of one bone wall (Figure 1) or even for an intact alveolus. It is based on alveolar occlusion, using a fixed provisional prosthesis with a specially designed pontic to provide stability to the coagulum and mechanical support to the gingival margin, preventing mucoperiosteal collapsing. There are some prerequisites when performing PDAR: teeth neighboring the compromised tooth area should be healthy and without proximal bone loss, and in esthetic areas, the gingival margin should be in harmony with the surrounding teeth and its contralateral counterpart. PDAR has a number of advantages, such as preservation of the preexisting alveolar bone by avoiding realizing incisions and flaps; regeneration of lost alveolar bone without the use of grafts or membrane barriers; and new bone formation in the space left by the tooth root using a minimally invasive procedure.

\section{Case Presentation}

In this clinical case, the first upper right pre-molar presented recurrent abscess in the buccal aspect, associate to the failure of a paraendodontic surgery performed 6 years ago (Figure 2). To perform the PDAR technique, a provisional fixed prosthesis (which can be adhesive) was created using a working cast. The gingival margin of the tooth to be removed (Figure 3) was marked with a $0.5 \mathrm{~mm}$ tip pencil. Then, the compromised tooth was removed from the cast and the "subgingival" area was excavated by approximately $3 \mathrm{~mm}$ (Figure 4), preserving the gingival margins of the area previously marked in pencil, creating a cylindrical niche. The pontic was then created, completely filling the excavated subgingival area. Subsequently, a $1 \mathrm{~mm}$ high and deep concavity was made in the intermediate subgingival millimeter, giving the subgingival area an hourglass shape (Figure 5). This concavity of the pontic is located subgingivally and will prevent gingival margin retraction. Ovoid pontics, by contrast, cause retraction of the gingival margin and failure of the procedure. The prosthesis disinfection should be performed by overnight immersion in $2 \%$ chlorhexidine solution. 


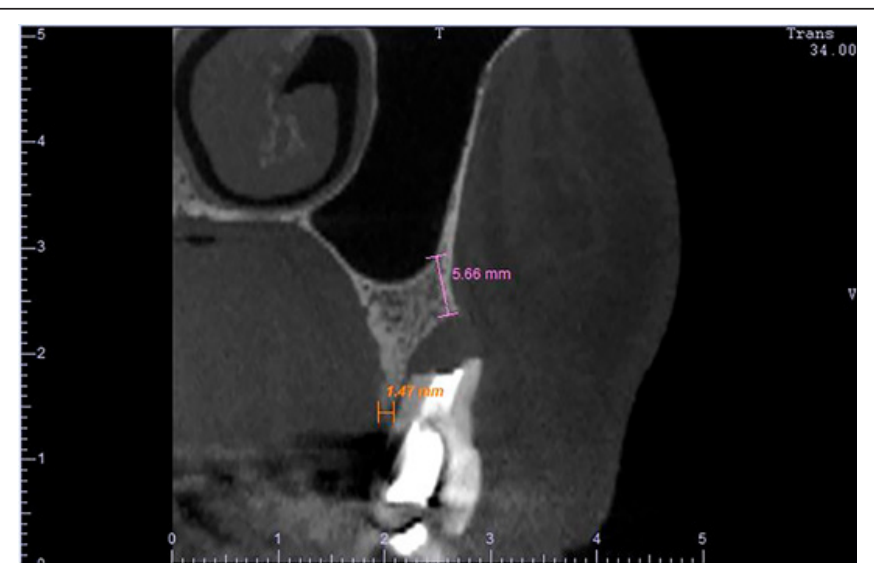

Figure 1: CBCT cross-sectional image of the tooth showing the complete loss of the buccal bone wall. The initial buccal bone height was of $5.66 \mathrm{~mm}$ (magenta) and the crestal bone thickness was of $1.47 \mathrm{~mm}$ (orange).

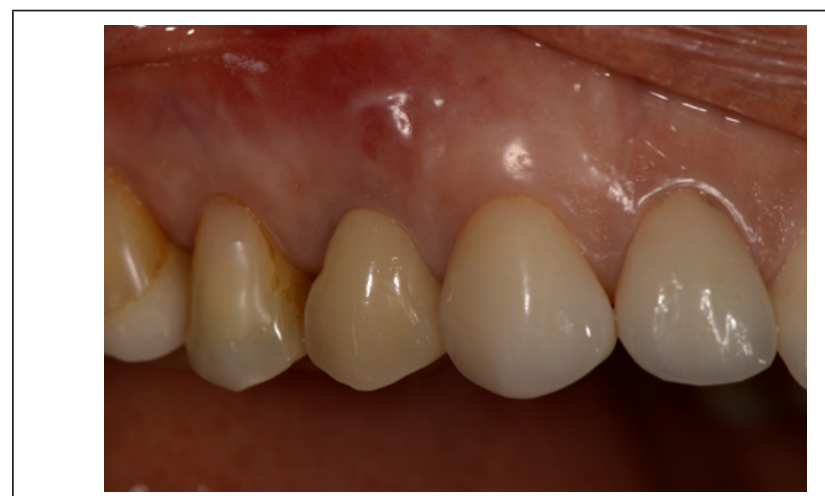

Figure 2: The mucosa presented redness and edema caused by an endoperiodontal abscess.

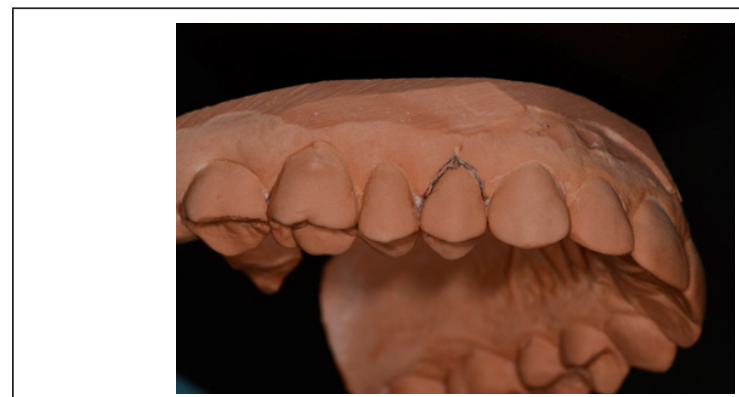

Figure 3: On the working cast the gingival margins were marked with a $0.5 \mathrm{~mm}$ tip pencil.

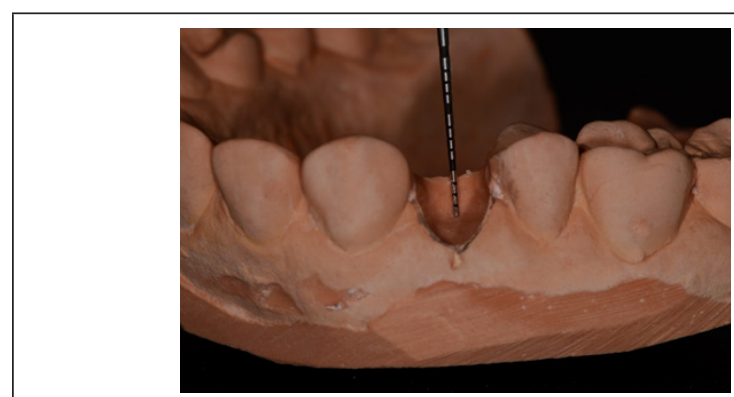

Figure 4: The tooth was removed, and a $3 \mathrm{~mm}$ subgingival niche was excavated in the working cast.
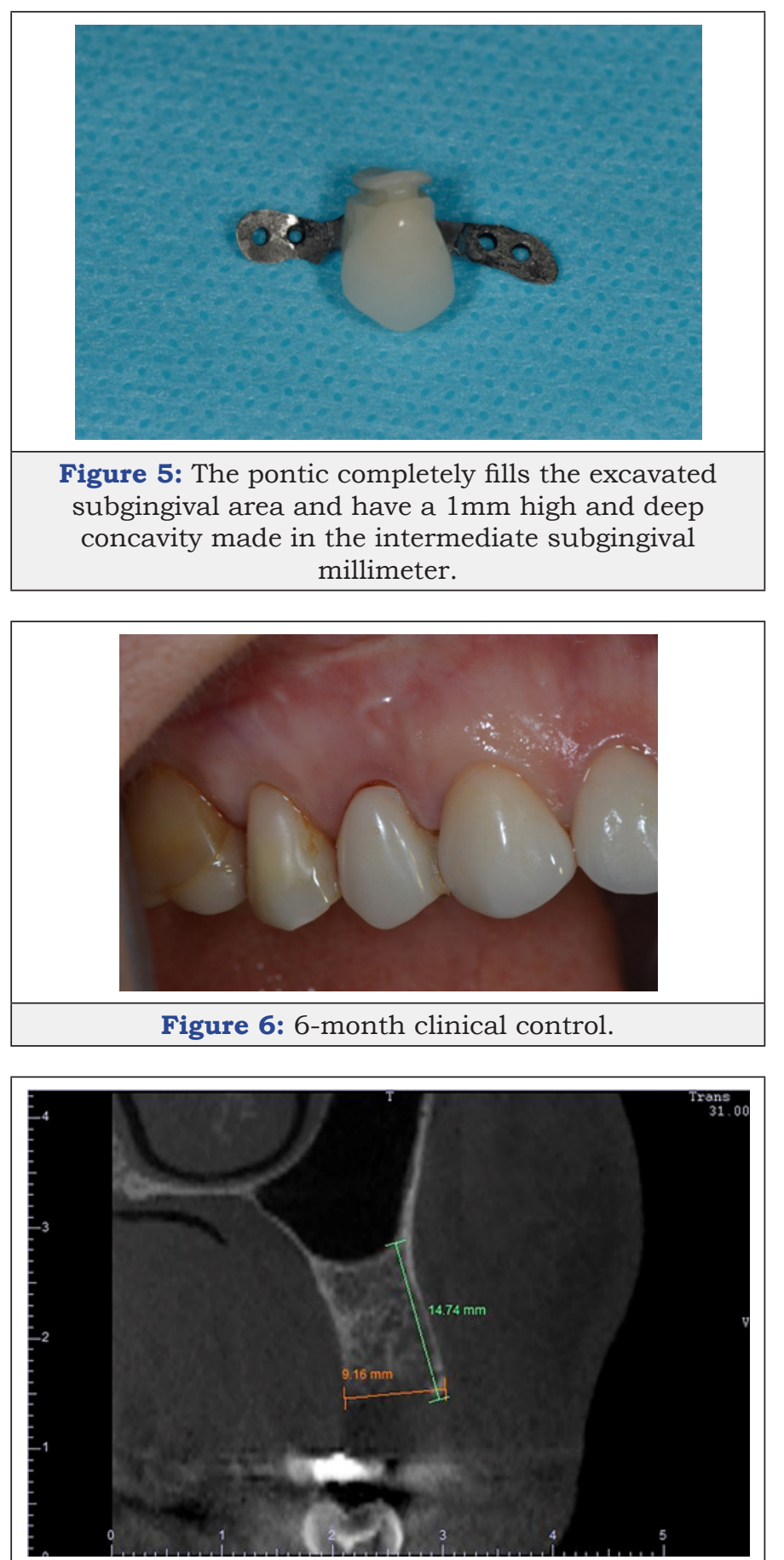

Figure 7: CBCT cross-sectional image shows a final buccal bone height of $14.74 \mathrm{~mm}$ (green) and a crestal bone thickness of $9.16 \mathrm{~mm}$ (orange) obtained without the use of grafts, membranes or flaps.

One hour before the surgery, the prosthesis should be immersed and kept in saline solution. It is extremely important that the whole subgingival area is rounded, with no sharp angles, well-polished and not over contoured, as this will cause recession of the mucosal margin, which may cause the procedure failure. Extraction was performed carefully using periotomes, without performing relaxing incisions and flaps. Before installation, the provisional prosthesis was tested to avoid direct contact with the bone, which can prevent its settlement into position. The provisional prosthesis was installed 
just after the tooth extraction using a fluid photopolymerizable resin ("Flow"). The patient was instructed not to floss in the pontic area. At the end of the healing period (Figure 6), after 6 months, a new cone beam computed tomography (CBCT) was performed to evaluate new bone formation (Figure 7).

\section{Discussion}

Traditional alveolar ridge regeneration techniques involve more invasive procedures with greater morbidity for patients (edema, pain, bruising). Traditional bone regeneration techniques may also involve other surgical sites, such as the donor area, or also higher costs for involving the use of biomaterials, such as grafts and membranes. Thus, PDAR is a less-invasive alternative, with no need for grafts or membranes, resulting in complete reconstruction of the alveolar ridge with optimum aesthetic results.

\section{References}

1. Dantas LRF, Groisman M, Vidigal Jr GM (2013) Prosthetically-driven alveolar reconstructions - the next step. In: Complex situations on Implant Dentistry: specialized clinical solutions. Rossetti PHO, Bonachela WC (Eds.), VM Cultural Editora, São Paulo, Brazil, pp. 16-31. 